\title{
ROBERT DELORT
}

\section{Aux racines des idées que les Occidentaux se font de leur pouvoir sur l'environnement}

Je voudrais tout d'abord revenir sur quelques définitions en ce qui concerne le mot Herrschaft, qui évoque le Herr, le seigneur, la domination, la souveraineté, l'autorité, voire la puissance mais n'a pas tous les sens que possède en français le mot »pouvoir«. En effet, pour nous, francophones, le pouvoir est en général la capacité de faire ou de faire exécuter une action particulière, en disposant des moyens nécessaires. Le pouvoir temporel est par exemple aux mains des politiques, des gouvernants qui dirigent, qui peuvent imposer des règles, donner et faire respecter des ordres. Si nous reprenons les distinctions classiques de Montesquieu, on peut évoquer le pouvoir de ceux qui font des lois; de ceux qui jugent les crimes et les différends des particuliers; de ceux qui exécutent les résolutions publiques. Ajoutons entre autres le fondamental pouvoir spirituel ou moral... et la fameuse dictature de la persuasion, chère aux admirateurs de Périclès comme à ceux sensibles aux beaux discours, à tous les moyens »médiatiques«, du livre et de l'image à la Toile, au Net.

De ce fait, j'hésite entre bien d'autres traductions du mot "pouvoir« en allemand classique: Fähigkeit, Gewalt, Kraft, Macht, Einfluß, Befähigung, Erlaubnis, Möglichkeit« (et, pourquoi pas, Regierung)? Sans compter les nuances plus ou moins subtiles entre les »können, sollen, mögen« et »dürfen«...

Bien entendu, tous ces termes peuvent être associés au mot »environnement« mais là nous avons la chance que Umwelt corresponde presque exactement à la définition récente dans l'Union européenne (1991) de »l'ensemble des éléments qui forment, dans la complexité de leurs relations, les cadres, les milieux et les conditions de vie de l'homme en société «1, c'est-à-dire moins que la umgebende Außenwelt de la célèbre formule de Haeckel (1866)2 qui évoque tout ce qui entoure un organisme quelconque, et pas encore la Mitwelt qui ne considère pas l'homme comme le centre obligatoire de la nature.

Finalement c'est avec quelque arbitraire que je vais envisager le pouvoir (de l'homme) sur l'environnement, comme Macht, autant que Herrschaft sur la Umwelt et reprendre quelques idées que j'ai plusieurs fois tenté de préciser, sans aborder les aspects purement politiques, technologiques, scientifiques, sentimentaux, magiques... ou autres.

Ce qui m'a toujours frappé, c'est comment les Occidentaux ont pu pousser jusqu'à ce point leur pouvoir sur la nature. Toutes les civilisations, bien entendu, ont eu des

1 Un certain nombre des idées exprimées ici proviennent de Robert DELORT, François WALTER, Histoire de l'environnement européen, Paris 2001, en particulier p. 19-20.

2 Cf. ibid., p. 20-21 avec in extenso la célèbre formule de Haeckel en allemand (et traduction commentée) et sa référence exacte. 
relations avec leur environnement. Mais il semble que l'Occident a, ici, une position spéciale puisqu'on dit bien que la domination sur le monde a été le fait de l'Occident à partir de la Renaissance et des grandes découvertes, et s'est pleinement développée du $\mathrm{XVI}^{\mathrm{e}}$ au $\mathrm{XX}^{\mathrm{e}}$ siècle. Quand je dis Occident c'est nous, Européens de l'Ouest, mais également nos frères russes et nos enfants américains, c'est-à-dire cet ensemble de civilisations que l'on dit occidentales. Cette domination de la nature, il faut bien le souligner, est du même type, qu'elle soit accomplie de la part des Soviétiques ou du côté des »capitalistes«. Je vais reprendre la plaisanterie de Coluche mais qui est très vraie: »Le capitalisme c'est l'exploitation de l'homme par l'homme; le communisme, c'est exactement le contraire«. Et, de fait, les Soviétiques au moins autant que les capitalistes ont considéré que la nature était au service de l'homme et même Gorki a signalé qu'»il faut créer une deuxième nature, c'est-à-dire une nature édifiée sur le terrain, sur les forces et les trésors de la première nature en organisant la nature antique, inorganisée et même hostile aux intérêts de l'humanité laborieuse«. C'est-à-dire que Gorki disait ce que le régime soviétique ou que les régimes capitalistes ont essayé de faire, d'exploiter la nature au maximum au profit de l'homme. Mais comment et pourquoi semblent-ils avoir réussi?

Tout le monde connaît le célèbre ouvrage américain, couronné par le prix Pulitzer (1998), qui s'appelle »Guns, Germs and Steel: the Fates of the Human Societies«. Son auteur, Jared Diamond, vient d'essayer de le reprendre ou de l'amplifier dans »Collapse: How Societies Choose to Fail or Succeed«, très proche de l'aussi récent (2006) »La fin du progrès«, du Canadien Ronald Wright et de bien d'autres. On reconnaît assez aisément, à propos de la Nouvelle Guinée, de l'île de Pâques ou de l'Amérique précolombienne que des sociétés se sont >effondrées` par surexploitation de leur environnement mais aussi que les Européens ont pu grandement en aider à s'effondrer parce qu'ils avaient des armes, des armes à feu, des germes qu'ils répandaient dans des milieux vierges, la variole a abattu les civilisations aztèques puis incas, et finalement l'acier, puisque les Européens y ont créé des armes et des instruments efficaces en grand nombre dans leurs aciéries, ce qui leur a permis d'asservir victorieusement (jusqu'à présent) leur propre environnement, et la planète Terre dans son ensemble. On peut à la rigueur envisager le comment. Mais qu'en est-il du pourquoi?

Il peut y avoir quelques raisons qui sautent aux yeux. Nous pouvons suivre en partie Lynn White, c'est lui qui, après beaucoup d'autres écrivains français, allemands ou anglais, a signalé que c'était le christianisme qui probablement avait amené - et il semble bien avoir raison de ce point de vue - les descendants d'Adam à dominer la nature, puisque c'est Dieu qui a donné à Adam cette domination. On connaît les termes de la Genèse: »Que l'homme domine sur les poissons de la mer [...] sur toute la terre etc.«. En fait l'homme après la Faute a bien été puni un petit peu puisqu'il doit travailler à la sueur de son front; n'empêche qu'il a été placé et maintenu à la tête de la Création. Et que effectivement, dans notre idéologie à l'heure actuelle nous exploitons la nature en étant persuadés que nous en sommes les maîtres. Je sais bien qu'il commence à y avoir de timides protestations de la part des Verts et d'autres personnes qui ne sont pas toujours au courant, mais il y a actuellement cette idée judéo-chrétienne (au moins implicite) ou cette persuasion atavique que l'homme est à la tête de la nature et doit 
l'exploiter. Cela dit, on peut aussi se demander pourquoi l'Occident a accepté cette vue (fondamentalement chrétienne) et bien différente de celle de nombreuses autres civilisations, de la domination de la nature?

Si on remonte avant le christianisme, on retrouve chez les Grecs en particulier, des idées qui concernent, bien sûr, des connaissances sur la nature, mais peu d'incitations philosophiques qui tendent à permettre, à Hésiode par exemple, l'exploitation pratique de son environnement. D'où ont pu naître de telles idées? Il y a au moins deux possibilités. La première, on l'a souvent évoquée, pourrait provenir du seul milieu occidental, qui s'est formé après la dernière glaciation, et jouissait (!) d'un climat tempéré, de beaucoup de feuillus, dont les feuilles formaient un humus fécond sur une terre profonde et des formations argilo-glaciaires, sans méconnaître des ressources minières provenant de l'érosion des montagnes hercyniennes et exploitables grâce au bois des forêts, etc. Il n'est pas impossible que l'environnement suggérait à 1 homme occidental des manières de l'exploiter plus évidentes que celles fournies par les milieux exubérants des zones tropicales ou les régions quasi-stériles des hautes latitudes ou circumpolaires...

Mais il y a peut-être autre chose. Et quand on regarde les débuts de la civilisation occidentale, la formation de l'Occident s'est réalisée sous trois influences: les chasseurs-cueilleurs depuis le Paléolithique, les agriculteurs-éleveurs du Néolithique, et puis aussi cette influence qui n'a peut-être pas été portée par les hommes, mais enfin qui a eu une importance fondamentale au moins sur les langues et les mentalités. D'où la question: dans un environnement déjà exploité, au moins partiellement, qu'ont pu apporter les »Indo-Européens«?

On ne sait pas s'il s'agissait de peuples, ou de simples influences, de peuple à peuple; les archéologues ont du mal à en retrouver des traces matérielles caractéristiques mais l'influence a apparemment existé puisque est attestée une langue (que l'on a pu recréer), langue qui est la mère de nos langues actuelles en Occident. Il faut alors regarder de nos jours les gens qui parlent ces langues et par quoi ils se distinguent des autres. Ils se distinguent de trois manières différentes. Si on remonte aux premiers millénaires, c'est à ce moment que l'on découvre le maniement du chiffre et que l'on s'y familiarise en comptant les troupeaux, le nombre de têtes, l'ampleur des stocks. Mais d'autres civilisations, parlant d'autres langues, semblaient les avoir largement précédées dans ces domaines et il est plus évocateur de tenter une étude de la langue elle-même et de celles qui en sont issues. Au $\mathrm{V}^{\mathrm{e}}$ siècle, en Grèce, on étudie certes la langue et la philologie et bien sûr la logique, mais il y a un autre endroit où, à la même époque, on étudie également la langue, la philologie et la logique, c'est l'espace indien autour de Panini pour purifier, la fameuse langue des Védas, le sanskrit, langue particulièrement proche de celle des cousins grecs.

On peut se demander s'il n'y a pas quelque chose dans les structures linguistiques et mentales des gens qui parlent ces langues, qui sont des langues à flexion, lesquelles, comme l'a écrit le regretté Pierre Lévêque, représentent »un outil intellectuel très performant en comparaison avec les langues agglutinantes antérieures, par les qualités intellectuelles qu'il requiert et développe à la fois « ${ }^{3}$. Ces langues, non seulement facili- 
tent la compréhension des choses, mais en même temps, elles permettent de comprendre plus loin... Cette réflexion est certes une attitude de linguiste. Mais on peut la rapprocher aussi d'une autre réflexion d'un grand linguiste, le regretté André Martinet qui considère une relation entre les structures mentales et les structures linguistiques. Dans un ouvrage au titre »environnementaliste«, »Des steppes à l'Océan« (1987), il écrit: »La conquête du monde, par les peuples de langues indo-européennes, qui ont su jusqu'ici garder une avance dans la mise de supériorités techniques au service de la violence, a commencé par la subjugation des populations préexistantes de l'Inde à l'Irlande. Elle ne s'est pas terminée avec la conquête de l'Ouest américain et de l'Asie septentrionale et l'impérialisme colonial [...]«. Doit-on en conclure que ces langues à flexion ont favorisé entre autres la violence mais aussi les »supériorités techniques«? Or il semble évident que le grand apport de la civilisation occidentale, comme l'a dit Valéry est bien »la création de la science« ${ }^{4}$. Et cette science fondée sur la logique et la mathématique semble bien n'être née qu'en Grèce et en Inde. Durant le Moyen Âge, par exemple, les Indiens nous apprennent le zéro, la numération de position, les chiffres, le tout transmis par le monde musulman, et surtout par des savants persans de langues indo-iraniennes utilisant l'arabe comme langue véhiculaire. On peut donc se demander si ces langues très apparentées ne nous ont pas permis d'une part de mieux nous attacher à l'étude de la nature et d'autre part de la mieux comprendre.

Si nous ajoutons (et pourquoi pas?) que ces langues indo-européennes ont entretenu l'agressivité de tous les points de vue: agressivité par rapport aux civilisations voisines, ce qui nous semble vrai, et aussi par rapport à la terre, surtout avec la culture du blé qui demande beaucoup de temps et beaucoup de force pour permettre les récoltes (et les défrichements), on voit s'esquisser une étonnante hypothèse qui peut se renforcer par la constatation que depuis le $\mathrm{XVI}^{\mathrm{e}}$ siècle tous les savants qui ont amené la domination du monde, en favorisant le développement de la physique et de l'ensemble des technologies de notre civilisation sont tous nés en Occident, en Angleterre, en France, en Allemagne, en Italie, en Russie ou plus récemment ont émigré surtout en Amérique du Nord ou sont nés aux États-Unis, mais toujours dans le concert de ces gens qui parlent ces langues à flexion, ces langues indo-européennes.

Les peuples nés de l'union de la douce Europe venue de Phénicie et du sauvage roi des dieux indo-européens Zeus pater se sont accrochés à une terre féconde (pourvu qu'elle fût travaillée par des efforts soutenus) et ont développé, plus ou moins agressivement, les possibilités mentales ou spirituelles que leur offraient les structures de leurs langues et la religion (venue comme leur mère du Proche-Orient) qu'ils pratiquaient pendant des siècles. Cette hypothèse combine la linguistique, la grammaire, l'histoire, les données de l'environnement ou de la théologie et pourrait apporter une lueur sur le déroulement, dans les sociétés occidentales, de la progressive prise du pouvoir de l'homme sur son environnement.

4 Cette phrase célèbre de Paul Valéry est extraite de l'article »Caractères de l'esprit européen«, paru en 1924 et repris tel quel en prologue à l'ouvrage d'Hélène AHRWEILER, Maurice AYMARD, Les Européens, Paris 2000; cf. aussi DELORT, WALTER, Histoire de l'environnement (voir n. 1), p. 34. 